\title{
Automatic Road Extraction from Satellite Imagery Using LEGION Networks
}

\author{
Jiangye Yuan, DeLiang Wang, Bo Wu, Lin Yan, and Rongxing Li
}

\begin{abstract}
We present an automatic method for road extraction from satellite imagery. The core of the proposed method is Locally Excitatory Globally Inhibitory Oscillator Networks (LEGION). We decompose the road extraction task into three stages. The first stage is image segmentation by LEGION. In the second stage, we compute the medial axis of each segment and select the segments with narrow widths. The third is the road grouping stage. With the medial axes, alignment-dependent connections between medial axis points are established and LEGION is utilized to group the well-aligned medial axes, which represent extracted road segments. Due to the selective gating mechanism of LEGION, different roads in an image are grouped separately. Experimental results on synthetic and real images show the effectiveness of this method.
\end{abstract}

\section{INTRODUCTION}

$\mathrm{D}$ ue to its utility for a variety of applications, road extraction from digital imagery has been intensively studied in the computer vision and remote sensing fields. Many techniques have been proposed, which can be broadly classified as semi-automatic approaches and automatic approaches. The main criterion for the classification is whether the approach requires human intervention [3], [11]. In semi-automatic approaches, an operator provides information such as starting points or starting directions, which provide critical assistance in tracking roads [10]. Without human intervention, an approach is considered automatic [2], [16].

Objects appear in natural scenes as groups of similar sensory features [19]. Gestalt psychology reveals a set of principles guiding the grouping process based on local features. Elements tend to be perceptually grouped if they are close to each other (proximity), similar to one another (similarity), form a smooth and continuous curve (good continuation), or have similar temporal behaviors (common fate) [14]. Since roads in satellite imagery tend to have uniform features that are distinct from neighboring regions, it is reasonable to expect that they can be automatically extracted using Gestalt grouping principles.

Dynamical systems represent a promising approach to object segmentation [8]. In particular, Locally Excitatory

Manuscript received December 9, 2008. This work was supported in part by an NGA University Research Initiatives grant (HM 1582-07-1-2027).

J. Yuan and D. L. Wang are with the Department of Computer Science \& Engineering and the Center for Cognitive Science, The Ohio State University, Columbus, OH 43210 USA (e-mail: yuanj@cse.ohio-state.edu; dwang@cse.ohio-state.edu).

B. Wu, L. Yan, and R. Li are with the Mapping and GIS Laboratory, the Department of Civil and Environmental Engineering and Geodetic Science, The Ohio State University, Columbus, OH 43210 USA (e-mail: wu.573@osu.edu; yan.351@osu.edu; li.282@osu.edu)
Globally Inhibitory Oscillator Networks (LEGION) proposed by Terman and Wang provides a framework to group features of an object and segment different objects through oscillatory correlation [15], [18], [19]. The oscillatory correlation theory asserts that oscillators corresponding to the pixels of the same object synchronize, and the oscillators corresponding to the pixels of different objects desynchronize. It has been shown that a LEGION network building on relaxation oscillators can rapidly achieve synchronization within a locally coupled oscillator assembly and desynchronization between different assemblies. LEGION has been successfully applied to a number of scene analysis tasks, including image segmentation, object selection, and speech segregation [17], [19].

In this paper, we present a new automatic approach to road extraction using LEGION dynamics. This approach is based on the observation that the roads are relatively thin and homogeneous regions with smooth directional changes. Our method has three stages: 1) image segmentation using a LEGION network; 2) medial axis extraction within each segment and selection of potential road segments; 3) grouping of potential road segments using a LEGION model with alignment-dependent connections based on extracted medial axis points. Here, well aligned segments are considered as belonging to the same road.

The rest of the paper is organized as follows. Section II briefly reviews the architecture and dynamics of LEGION. In Section III, we present our method including image segmentation, candidate segment selection, and road segment grouping. Section IV reports experimental results, and Section $\mathrm{V}$ gives conclusion and further discussion.

\section{LEGION MODEL}

LEGION is a network of Terman-Wang oscillators [8]. An oscillator $i$ is typically defined as a feedback loop between an excitatory unit $x_{i}$ and an inhibitory unit $y_{i}$ :

$$
\begin{aligned}
& \dot{x}_{i}=3 x_{i}-x_{i}^{3}+2-y_{i}+I_{i}+S_{i}+\rho \\
& \dot{y}_{i}=\varepsilon\left(\alpha\left(1+\tanh \left(x_{i} / \beta\right)\right)-y_{i}\right)
\end{aligned}
$$

where $I_{i}$ denotes external stimulation, $S_{i}$ overall internal input from other parts of the network, and $\rho$ is a noise term. The variable $\varepsilon$ is set to be a very small positive number. In this case, (1) defines a typical relaxation oscillator, as illustrated in Fig. 1. The $x$-nullcline of the oscillator is a cubic curve, and the $y$-nullcline is a sigmoid function. When $I_{i}>0$, these curves intersect along the middle branch of the cubic and the oscillator is enabled (Fig. 1a). When $I_{i}<0$, the oscillator approaches a fixed point (Fig. 1b); in this situation the 
oscillator is in an excitable state. An excitable oscillator could be enabled if it receives large enough overall input $S_{i}$. When the oscillator becomes oscillatory, its periodic orbit alternates between a silent phase, corresponding to low $x$ activity, and an active phase, corresponding to high $x$ activity. The dynamic evolution of the oscillator proceeds slowly within the silent or the active phase, while the alternation between the two phases occurs on a fast time scale, referred to as jumping. $\alpha$ is the parameter to control the relative durations of the two phases. $\beta$ determines the steepness of the sigmoid (see Fig. 1).

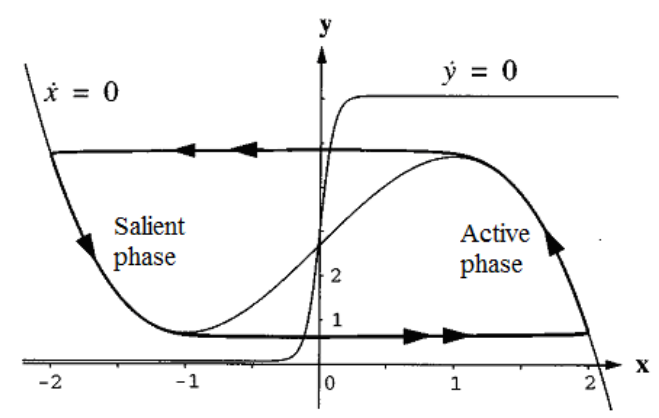

(a)

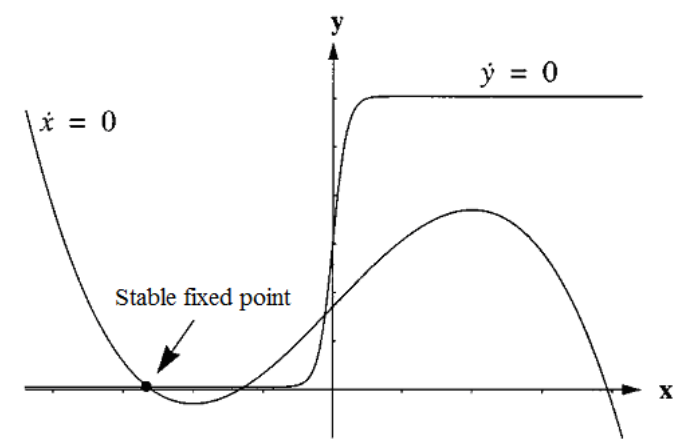

(b)

Fig. 1. Dynamics of a single oscillator. (a) Behavior of an enabled oscillator, which produces a limit cycle trajectory. The arrows indicate the motion direction. (b) Behavior of an excitable oscillator. In this case, it approaches a stable fixed point.

The simplest LEGION network is designed as a two-dimensional (2-D) grid, as shown in Fig. 2. An oscillator is connected to its four nearest neighbors, where the connections are all excitatory. A global inhibitor receives excitation from each oscillator in the network and then inhibits each oscillator. The coupling term $S_{i}$ in (1a) is defined as follows

$$
S_{i}=\sum_{j \in N(i)} W_{i j} H\left(x_{j}-\theta_{x}\right)-W_{z} H\left(z-\theta_{z}\right)
$$

Here, $W_{i j}$ is a connection weight that determines the amount of excitation oscillator $j$ sends to oscillator $i$, and $N(i)$ is the set of the neighboring oscillators that connect to $i$, the size of which can be chosen differently for specific tasks. $H$ is the Heaviside step function. Both $\theta_{x}$ and $\theta_{z}$ are thresholds. An oscillator sends excitation to its neighbors, and the global inhibitor exerts inhibition only when its activity exceeds a certain threshold. $W_{z}$ is the weight of inhibition from the global inhibitor $z$, whose activity is given by

$$
\dot{z}=\phi\left(\sigma_{\infty}-z\right)
$$

where $\phi$ is a parameter, and $\sigma_{\infty}$ equals one if $x_{i} \geq \theta_{x}$ for at least one oscillator and $\sigma_{\infty}$ equals zero otherwise.

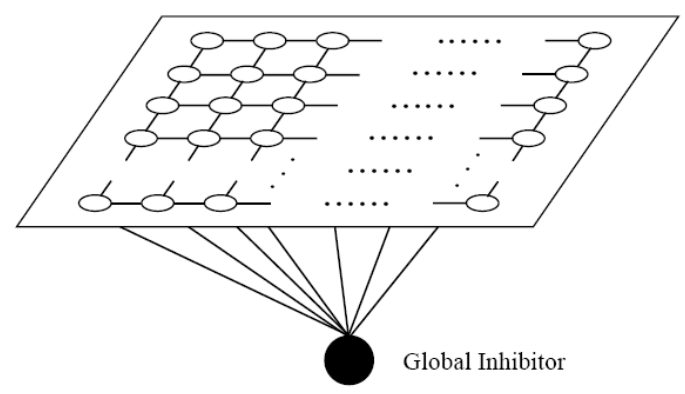

Fig. 2. Architecture of two-dimensional LEGION network.

The basic dynamics of LEGION can be briefly described as follows. Once an oscillator is in the active phase, it triggers the global inhibitor, which attempts to inhibit the entire network. The inhibition prevents the oscillators of different assemblies from jumping but does not affect the oscillators of the same assembly because of local excitation. An enabled oscillator propagates its excitation to its neighbors and from them to its further neighbors until all the oscillators of the same assembly are enabled. Thus, the dynamics of LEGION is a process of both synchronization and desynchronization, achieved respectively by local cooperation through excitatory coupling and global competition via the global inhibitor [15].

\section{LEGION BASED ROAD EXTRACTION}

This section describes a new method for road extraction. After LEGION-based image segmentation, the medial axes of segments are extracted and road segment candidates are selected based on segment widths. By establishing the alignment-dependent connections between medial axis points of candidate segments, LEGION is further used to group the medial axes representing road segments. The final result of road extraction is obtained based on the grouped medial axis points.

\section{A. Image segmentation using LEGION}

LEGION has been successfully applied to gray-level image segmentation [4], [5], [19]. For processing a 2-D image, each oscillator in a network corresponds to a pixel of the image and is connected to its eight nearest neighbors. The connection weight between two adjacent oscillators is set to be proportional to the similarity of the corresponding pixel gray values.

When handling real images, image noise can cause fragmentation, which impairs LEGION segmentation. To address this problem, a lateral potential for each oscillator is introduced [19]. The idea is to distinguish a major oscillator block that contains at least one oscillator (called a leader) located at the center of a large homogeneous region, while a 
noisy fragment does not produce a leader. A leader receives large excitation from its neighborhood, hence its high lateral potential. In the segmentation process, leaders are stimulated to enable oscillator groups. All the fragments correspond to oscillators that cannot sustain oscillations and are hence considered as background. Moreover, to facilitate computation, an abstracted algorithm following the main steps of LEGION dynamics has been developed. Detailed description of this algorithm and its extended version can be found in [5], [19].

Roads in a digital image appear as thin and elongated homogeneous regions. Since leaders are required to be at the center of the large homogeneous regions, roads rarely contain leaders and thus are mostly segmented to the background. For example, Fig. 3(b) shows a segmentation result of the image in Fig. 3(a), in which roads are put into the background, indicated by black areas. For obtaining road segments, a region growing process is adopted following LEGION segmentation. We treat each background pixel as a starting point and perform pixel-based growing, which repeatedly checks the neighbor of the starting point and adding it into the region if it is similar to the starting point. If a pixel can grow into a large enough area, the area is considered as a new segment. Fig. 2(c) shows the result after the subsequent step, where road segments are attained. It should be noticed that a road cannot be segmented as a single segment for most real images.

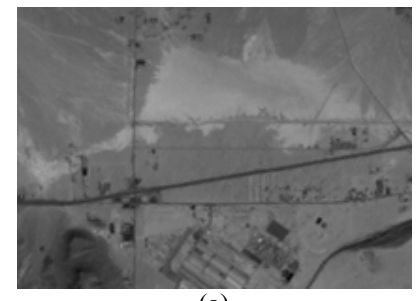

(a)

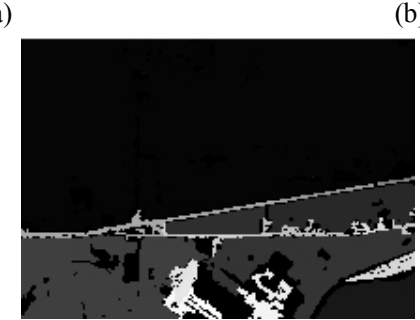

(c)

Fig. 3. LEGION image segmentation. (a) A satellite image containing roads. (b) The result of LEGION segmentation where each gray level indicates a distinct segment. (c) The result after a subsequent step for road extraction.

\section{B. Medial axis transform and candidate segment selection}

After we obtain the segmentation of an image, we seek to select the segments that correspond to roads. The medial axis transform is employed to achieve this goal. The concept of the medial axis transform is to transform a two-dimensional object into a one-dimensional line representation that largely preserves the extent and connectivity of the original object. The medial axis of an object is defined as the loci of the centers of all the maximally inscribed circles of the object. The radius of the circle, i.e., the shortest distance to the boundary, recorded at a point of the medial axis provides information about local thickness.
A major approach to compute the medial axis is the Voronoi diagram [1], [6], [12]. Given a set of points $S$ in a plane, the Voronoi diagram is a partition of the plane into cells, each of which contains all the points in the plane closer to one particular point in $S$ than to any others. It has been proven that the vertices of the Voronoi diagram for a set of samples on a boundary curve in the object approximate the medial axis. The more densely the space is sampled, the more accurate the medial axis is, as shown in Fig. 4.

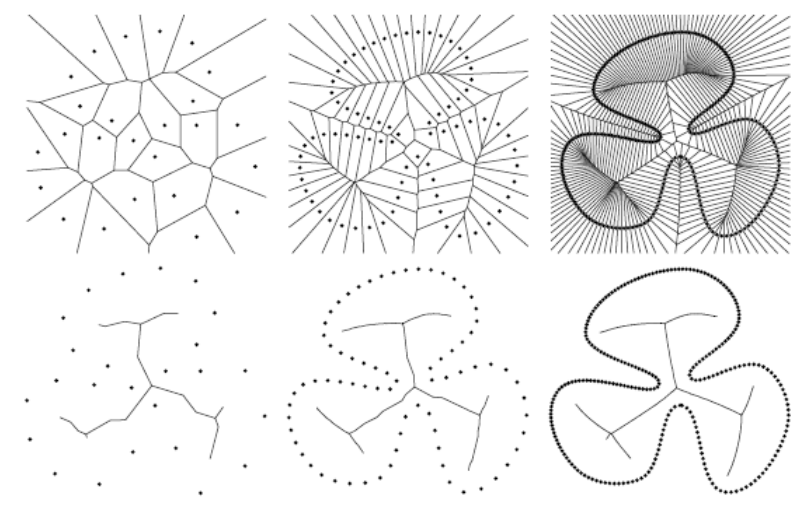

Fig. 4. Medial axis approximated by the Voronoi diagram. In each column, the top is the Voronoi diagram for a set of samples on the boundary, and the bottom is the Voronoi vertices and edges lying inside the shape. (from [1]).

By treating the boundary pixels of segments as the samples, the medial axis points for each segment are obtained from the Voronoi diagram. Since road segments are characterized by their narrow widths, a segment is selected as a candidate segment if the average distance of medial axis points of the segment to its boundary is sufficiently small.

\section{Road segment grouping using LEGION}

Selected segments by the medial axis transform mostly correspond to roads. However, there are non-road segments that are also selected due to their thin shapes. In order to accurately group segments to form roads and eliminate non-road segments, we propose a LEGION model with alignment-dependent connections.

In an image containing oriented elements, some sets of elements tend to be perceptually grouped [21]. It has been suggested that this perceptual effect is mediated by long-range horizontal connections within the visual cortex, which are dependent on the positions and orientations between the elements [7], [9], [20]. For an oriented element, there exist two types of excitatory connections, one spreading along the axis of the element orientation, called co-axial, and another limited to a narrow zone orthogonal to the orientation axis, called trans-axial [21].

The co-axial connection is a pattern similar to the co-circular connectivity pattern [13], as shown in Fig. 5(a). For the element at location $A$ with local orientation $\theta$, there is a preferred orientation $\phi$ for the element at location $B$, which is determined by the following rule. Given orientation $\theta$ and locations $A$ and $B$, a unique circle exists which passes through $A$ and $B$ and has a tangent orientation at $A$ identical to $\theta$. 
Orientation $\phi$ is specified by the tangent to the circle at $B$, computed by the following equation

$$
\phi=2 \tan ^{-1}\left(\frac{y}{x}\right)-\theta
$$

where $(x, y)$ are the coordinates of $B$ with origin at $A$. If the local orientation at $B$, denoted by $\psi$, accords with the preferred orientation $\phi, A$ receives the strongest excitation from $B$. As $\psi$ deviates from $\phi$, the connection weight falls off following a Gaussian function. The connection weight also decreases as a Gaussian function of the distance between $A$ and $B$. Note that the co-axial connections are confined to low curvature deviations from the orientation axis.

For trans-axial connection, the preferred orientation of an element is parallel to that of the target element. This set of connections is more spatially sensitive. The weights decrease as the angle deviation increases much more quickly than those of co-axial connections.

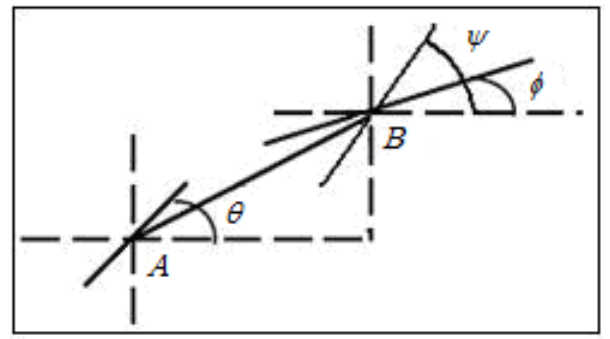

(a)

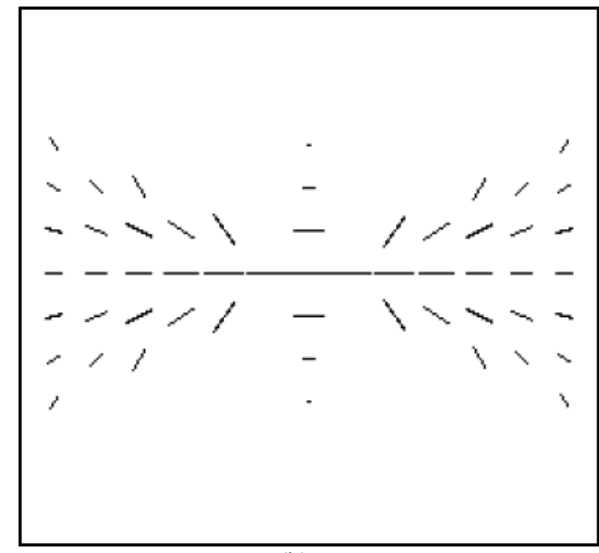

(b)

Fig. 5. Illustration of horizontal connections. (a) Co-circularity constraint. $\theta$ and $\phi$ are the tangents to the circle passing through $A$ and $B$. (b) Connectivity pattern of a horizontally oriented cell (from [21]). The cell is located at the center of the image. The orientations of lines represent the preferred orientations and the length of a line indicates connection strength.

The connectivity pattern of a horizontally oriented element is illustrated in Fig. 5(b), which shows the preferred orientations and connection strengths. The connection weight between two elements can be written as

$$
W=G\left(|\psi-\phi|_{\text {acute }}, \sigma_{\psi}\right) \times G\left(\sqrt{x^{2}+y^{2}}, \sigma_{d}\right)
$$

where $|\psi-\phi|_{\text {acute }}$ denotes the acute angle between $\psi$ and $\phi$, $G$ stands for a Gaussian function, and $\sigma_{\psi}$ and $\sigma_{d}$ are set differently for two different types of connections.

Under the assumption that road segments are aligned collinearly or curvilinearly, we apply a LEGION model with long-range horizontal connections to the medial axis points of segments to simulate the perceptual "pop-out" for well-aligned points and thus implement road segment grouping.

Medial axis points, which are available from the previous stage, are considered as oscillators. Due to the onedimensional nature of a medial axis, the orientation of each oscillator can be readily calculated from its neighborhood. The coupling term $S_{i}$ of oscillator $i$ takes the following form:

$$
S_{i}=\operatorname{Max}_{j \in N(i)}\left\{W_{i j} H\left(x_{j}-\theta_{x}\right)\right\}-W_{z} H\left(z-\theta_{z}\right)
$$

where $W_{i j}$ is the weight given by the long-range horizontal connection. The neighborhood of oscillator $i$ is set as a window centered at $i$ and it is larger than that in the image segmentation stage. Here, instead of summation, a maximization operation is adopted, which focuses on the pairwise relations of oscillators. The maximization operation ensures that each oscillator, whether the corresponding medial axis point is in the middle or at the end of a road, has the same overall effective connection weight from its neighbors.

Similar to applying LEGION to image segmentation, leaders need to be selected and stimulated for the sake of avoiding fragmentation. By extending the concept of lateral potential, we observe that the oscillators corresponding to the medial axis of a segment with a long, thin and smooth shape tend to have high lateral potentials. A simple method is used to identify leaders. For a candidate segment, a closing operation is first performed to smooth the boundary - closing is defined as a dilation operation where each background pixel next to an object pixel is changed into an object pixel, followed by an erosion operation where each object pixel next to a background pixel is changed into a background pixel. Then, the medial axis is computed. If the average distance between the boundary and the medial axis points is sufficiently small and the length of the medial axis is sufficiently large, then the corresponding oscillators become leaders.

By LEGION dynamics, oscillators corresponding to the medial axis points of one road are synchronized and those corresponding to the medial axis points of different roads are desynchronized. The oscillators that are not well aligned with any of the leaders remain excitable throughout the process. After obtaining the medial axis points representing road segments, a segment is extracted as a road segment if most of its medial axis points are activated, which completes the task of extracting road segments and removing noisy segments.

\section{EXPERIMENTAL RESULTS}

To illustrate the behavior of the LEGION network with long-range horizontal connections, we have simulated a 
$50 \times 50$ oscillator network. The input image is shown in Fig 6(a). There are three patterns, which consist of discontinuous but smoothly aligned points. These points can be considered as the medial axis points of different road segments.

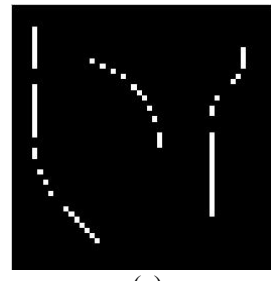

(a)

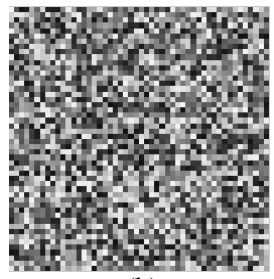

(b)

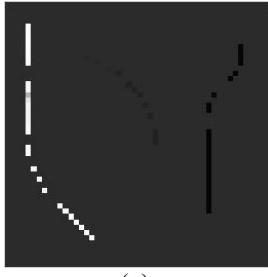

(c)

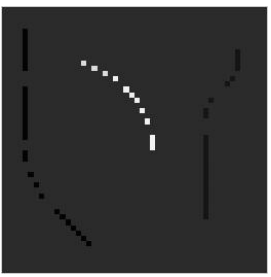

(e)
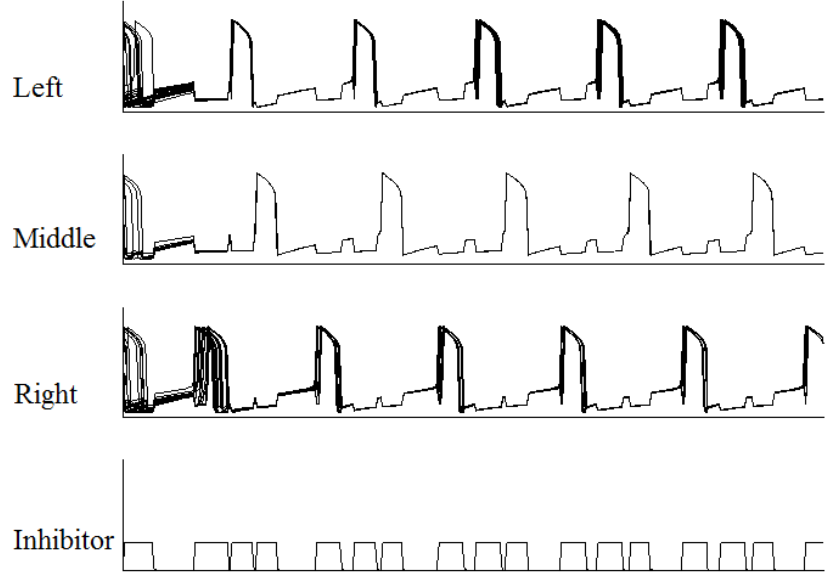

(f)

Fig. 6. Road segment extraction. (a) A 50×50 synthetic image containing three interrupted roads. (b) A snapshot at the beginning of dynamic evolution showing random phrases of the corresponding oscillators. (c)-(e) A sequence of snapshots after (b) showing extracted road segments. (f) The upper three traces show the combined $x$ activities of all the oscillators, where the oscillators representing the same pattern are combined in one trace. The bottom trace shows the activity of the global inhibitor.

In the simulation all oscillators corresponding to the object pixels are stimulated with $I=2.0$. The differential equations are solved using the fourth-order Runge-Kutta method with the following parameter setting: $\varepsilon=0.02, \gamma=5.0, \beta=0.1$, $K=50, \theta_{x}=-0.5, \theta_{z}=0.1$, and $\phi=30$. The orientation of each oscillator is approximated by the line that passes through the oscillator and its nearest neighbor. Fig. 6(b)-(e) shows the instantaneous activity of the network at various stages of dynamic evolution. The gray value of each pixel represents the $x$ activity of the corresponding oscillator. It can be seen that the oscillators representing each pattern reach their active phase and separate from the rest of the image. This successive "pop-out" of the patterns continues in a stable periodic way. Fig. 6(f) shows the temporal evolution of all the oscillators. The activities of the oscillators of each pattern are combined as one trace. The effect of synchrony and desynchrony can be clearly observed.

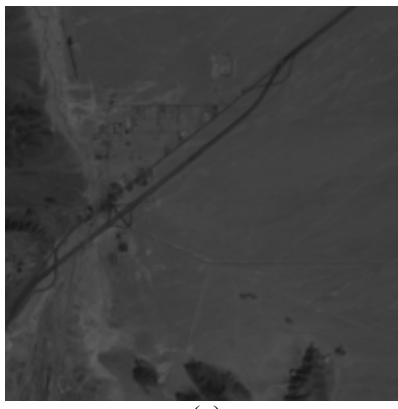

(a)

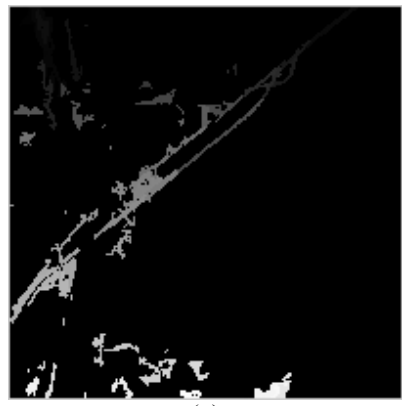

(c)

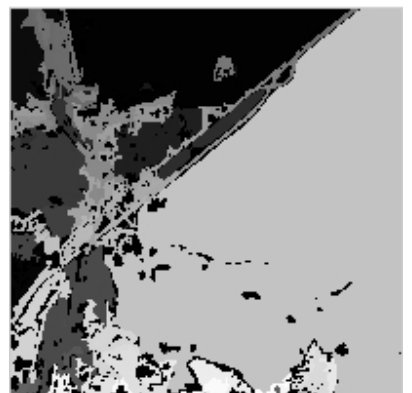

(b)

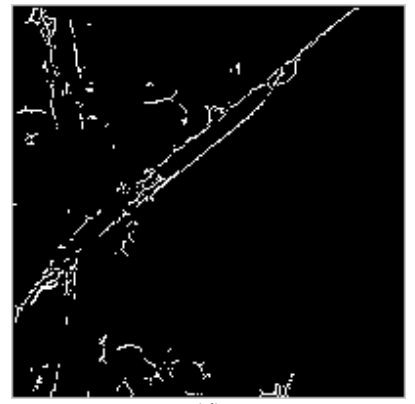

(d)

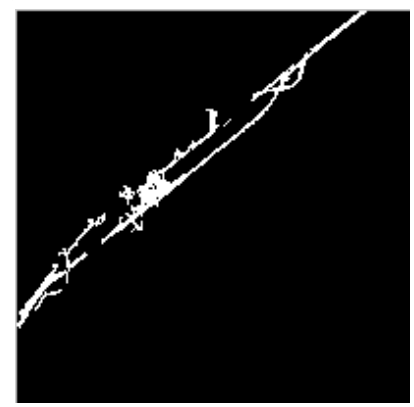

(e)

Fig. 7. Results of road extraction from an image. (a) A satellite image containing a road $(200 \times 200$ pixels $)$. (b) The result of LEGION segmentation. (c) Candidate segments. (d) The medial axes of candidate segments. (e) The final result of road extraction.

Next, we show the results of applying our method to real satellite images. To alleviate the computational burden when processing large real images, the abstracted algorithm of LEGION mentioned earlier is adopted in the first and third stages. The first image our method is tested on is shown in Fig. 7(a), which is a hyperspectral satellite image with a resolution of 30 meters collected at Silver Lake in the Mojave Desert, California. This image contains one road that has two main branches. Fig. 7(b) shows the segmentation result, where each gray level indicates a distinct segment. The candidate segments are then selected based on the segmentation result, shown in Fig. 7(c), and the medial axes of all the candidate segments are illustrated in Fig. 7(d). To facilitate computation, the medial axis points are moved to their nearest pixel locations. The final result is obtained by grouping the candidate road segments, shown in Fig. 7(e). Since no leader is generated among wrongly selected candidate segments, these segments are eliminated. We can see that the main part 
of the road, as well as several small branches, is successfully extracted.

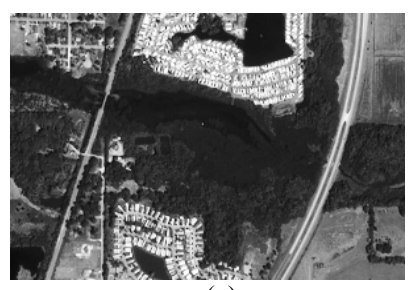

(a)

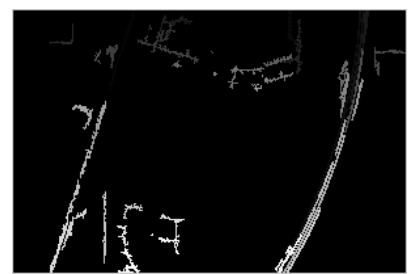

(c)

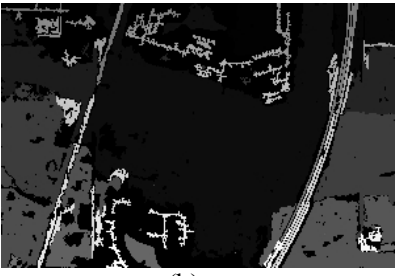

(b)

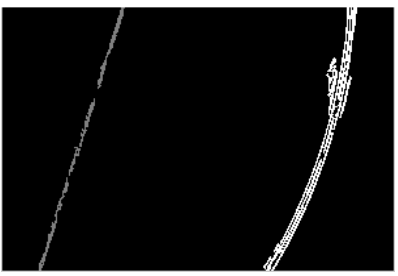

(d)
Fig. 8. Results of road extraction from another image. (a) A satellite image containing two roads $(300 \times 200$ pixels $)$. (b) The result of LEGION segmentation. (c) Candidate segments. (d) The final result of road extraction.

The next test image is another satellite image, which has a resolution of 4 meters collected at Tampa Bay, Florida. This image contains two major roads, as shown in Fig. 8(a). Fig. 8 (b)-(d) illustrates the results of the three stages of our method. In the third stage, two roads are produced separately, which are indicated by different gray levels. As can be seen, both roads are clearly extracted.

These as well as other results not shown demonstrate that our proposed approach for automatic road extraction is successful in extracting roads from satellite imagery.

\section{CONCLUSION AND DISCUSSION}

In this paper, we have presented a new automatic road extraction method based on LEGION. Three stages are used to implement road extraction: image segmentation, candidate segment selection, and road segment grouping. Simulation results confirm the effectiveness of this method.

The fundamental idea of the proposed method is grouping similar features and segregating dissimilar ones. In the first stage the gray level is chosen as a feature, which can be interpreted as similarity, and in the third stage orientation alignment is taken as a feature, which can be interpreted as good continuation. Both features are encoded in the LEGION model, which uses dynamics of oscillatory correlation to achieve grouping. The generality of the underlying idea implies that the method could be used in a wide range of applications where both region and boundary information need to be considered.

In the present study, we did not perform prior smoothing for images, a necessary step in many road extraction methods, because noisy fragment removal is incorporated in the LEGION segmentation process. However, smoothing can be used as preprocessing to improve the quality of resultant segments for certain imagery, and will be included in future work.

\section{REFERENCES}

[1] D. Attali, J.-D. Boissonnat and H. Edelsbrunner, "Stability and computation of the medial axis - a state-of-the-art report," In Mathematical Foundations of Scientific Visualization, Computer Graphics, and Massive Data Exploration, T. Möller, B. Hamann, and B. Russell, Ed, Springer-Verlag, 2007.

[2] M. Barzohar and D. B. Cooper, "Automatic finding of main roads in aerial images by using geometric stochastic models and estimation," IEEE Transactions on Pattern Analysis and Machine Intelligence, vol. 18(7), pp. 707-721, 1996.

[3] A. Baumgartner, C .Steger, H. Mayer, W. Echstein, and H. Ebner, "Automatic road extraction based on multi-scale, grouping, and context," Photogrammetric Engineering and Remote Sensing, vol. 65(7), pp. 777-785, 1999.

[4] K. Chen and D. L. Wang, "A dynamically coupled neural oscillator network for image segmentation," Neural Networks, vol. 15, pp. 423-439, 2002.

[5] K. Chen, D. L. Wang, and X. Liu, "Weight adaptation and oscillatory correlation for image segmentation," IEEE Transaction on Neural Networks, vol. 11, pp. 1106-1123, 2000.

[6] T. K. Dey and W. Zhao, "Approximating the medial axis from the Voronoi diagram with a convergence guarantee," Algorithmica, vol. 38, pp. 179-200, 2003.

[7] D. J. Field, A. Hayes, and R. F. Hess, "Contour integration by the human visual system: Evidence for a local 'Association Field'," Vision Research, vol. 33, pp. 173-193, 1993.

[8] C. Gros, Complex and adaptive dynamical systems: A primer. Springer, Berlin, 2008.

[9] I. Kovács, U. Polat, and A. M. Norcia, "Breakdown of binding mechanisms in amblyopia," Investigative Ophthalmology \& Visual Science, vol. 37, p. 3078, 1996.

[10] D. McKeown and J. Denlinger, "Cooperative methods for road tracking in aerial imagery," in Proc. Computer Vision and Pattern Recognition (CVPR), pp. 662-672, 1988.

[11] X. Niu, "A semi-automatic framework for highway extraction and vehicle detection based on a geometric deformable model," Photogrammetric Engineering and Remote Sensing, vol. 61, pp. 170-186, 2006.

[12] R. L. Ogniewicz and O. Kübler, "Hierarchic Voronoi skeletons," Pattern Recognition, vol. 28(3), pp. 343-359, 1995.

[13] P. Parent and S. W Zucker, "Trace inference, curvature consistency, and curve detection," IEEE Transactions on Pattern Analysis and Machine Intelligence, vol. 11, pp. 823-839, 1989.

[14] I. Rock and S. Palmer, "The legacy of Gestalt psychology," Scientific American, vol. 263, pp. 84-90. 1990.

[15] D. Terman and D.L. Wang, "Global competition and local cooperation in a network of neural oscillators," Physica D, vol. 81, pp. 148-176, 1995.

[16] G. Vosselman and J. de Knecht, "Road tracing by profile matching and Kalman filtering," in Proc. Workshop on Automatic Extraction of Man-Made Objects from Aerial and Space Images, A. Gruen, O. Kuebler, and P. Agouris, Ed, Birkhäuser Verlag, Basel, pp. 265-274, 1995.

[17] D. L. Wang, "Object selection based on oscillatory correlation," Neural Networks, vol. 12, pp. 579-592, 1999.

[18] D. L. Wang, "The time dimension for scene analysis," IEEE Transactions on Neural Network, vol. 16, pp. 1401-1426, 2005.

[19] D. L. Wang and D. Terman, "Image segmentation based on oscillatory correlation," Neural Computation, vol. 9, pp. 805-836, 1997.

[20] S.-C. Yen and L. H. Finkel, "Salient contour integration by temporal binding in a cortically-based network," in Proc. Neural Information Processing Systems (NIPS-96), pp. 915-921, 1997.

[21] S.-C. Yen and L. H. Finkel, "Extraction of perceptually salient contours by striate cortical networks," Vision Research, vol. 38, pp. 719-741, 1998. 